\title{
Mobile Application Implementing Location Based Services Framework with Google Cloud Platform Integration: SSRU Development Case
}

\author{
Shutchapol Chopvitayakun
}

\begin{abstract}
Finding a place in a new location where visitors have no experience and clueless about it. This seems like getting lost in a maze and there is no easy way to reach out destination. To solve this problem, Location Based Services (LBS) plays an important role in providing visitors with assistance, direction, guideline and other relevant information. With LBS, visitors will be given walking instructions to get to a desirable place correctly. Moreover, visitors are enabled to find out the best route to approach destination efficiently. Then, no one wastes time and gets lost. This work applied Location Based Services framework integrated with Google Maps Platform to develop a mobile application called SSRU Map, run on Android Operating System. This application provides a series of geolocation information and solution featured on the location of Suan Sunandha Rajabhat University (SSRU), Thailand. Each year, SSRU has thousand visitors come to this university with various purposes e.g. applicants for admission or seminar participants. SSRU geographical landscape is not a rectangular shape and it is not a grid layout either. That is why it is harder for visitors to locate a building they are looking for by themselves. This mobile application is devoted for the main campus of SSRU in Bangkok with its area around 2 square kilometers. Main features of this application cover all locations and routes of major and minor buildings on this campus. Besides navigation feature, it also provides each building information e.g. pictures of interior and exterior of each building, website information, and contact information. This application will serve thousand visitors every year while.
\end{abstract}

Index Terms-Android, Google Could, location based services, mobile application, SSRU.

\section{INTRODUCTION}

Suan Sunandha Rajabhat University (SSRU), Thailand is a public university located in downtown area of Bangkok capital city of Thailand. This university was a palace around one hundred years ago. Then, it is not just an academic institute but both archeological site and attraction stile as well. Each year, SSRU main campus in Bangkok, welcomes thousand visitors as roles of a test center, an attraction site and a host for variety events e.g. open-house, seminar, conference, and training. Most visitors are not familiar with SSRU topology due to it is not a block shape and a grid format. Most visitors get lost and waste a lot of time finding assistance. Campus maps are available online and offline. Online version is available on http://www.ssru.ac.th and offline versions are available at some entrance gates and

Manuscript received June 14, 2019; revised October 28, 2019. This work was supported by Suan Sunandha Rajabhat University.

Shutchapol Chopvitayakun is with Information Technology Program, Faculty of Science and Technology, Suan Sunandha Rajabhat University, Bangkok, Thailand (e-mail: shutchapol.ch@ssru.ac.th). some areas on campus. However, these maps are not much helpful for visitors. They are static graphics and visitors are required for map reading skill and map interpretation techniques. Then, most of the time, when visitors get lost, the best solution for them is finding a nearby personal assistant. Unfortunately, not every person knows every building and all the routes efficiently. Even getting help from a nearby person but it is not really helpful at all. Sometime, visitors are getting more confused, still lost and need more help. Then, Location Based Services (LBS) can solve these problems for visitors. This framework provides various services through computer software regarding SSRU location, routes, and places.

Navigation system plays an important role in exploring new places for visitors. Navigation delivered through Location Based Services (LBS) is a framework implemented in this project. Location-based services are defined as all kinds of service that make access to the geographic location of an object. This object could be human or non-human that trigger its location as a service [1]. These services integrate location or position retrieved from mobile devices. Moreover, there is other relevant information also provided not only location information to user. Location-based services can be seen as the convergence of mobile services, location aware technologies (positioning) with the Internet and Geographic Information System [2].

Google Maps Platform, previously known as Google Maps. Recently, Google Maps Platform just merged with Google Cloud Platform (GCP) to provide all Google services e.g. compute engine, cloud storage, and application engine through cloud service or internet. Google Cloud Platform instantly makes available for use of Application Programing Interface (API) for various types of software developer. There are many businesses and projects e.g. ride share, game, real estate, and travel that deploy Google Maps Platform on their background services. This work implemented Google API services, retrieved from Google Maps Platform integrated with Java Development Kit (JDK) to compile and build a native mobile application run on Android operating system.

\section{LITERATURE REVIEW}

Location Based Services systems (LBS) was investigated for its architecture characteristics, platforms, and technologies in order to combine those studied factors for the better results of a proposed system. For better performance of a new system, it will provide precision and reliability to its users [2].

There are 5 major components of Location Based Services: 
1) Mobile devices: it could be smartphone, computer or any smart gadgets with capability of navigation.

2) Communication Network: it is a connecting point for interchange of data and services. Mobile devices transmit and receive information from a network where they connect.

3) Positioning Component: It could be a chipset that features Global Positioning System (GPS) or communication network which it can pinpoint position of device. Moreover, Wireless access point and radio transmitter could provide position or location of device either.

4) Service and Application Provider: Services e.g. finding route or location information requested by users are delivered by service and application provider. It is not just services regarding location but other relevant and interesting information i.e. point of interest is provided as well.

5) Data and Content Provider: Geographic data is stored and maintained by a provider to ensure that it is available for everyone including authority users and business partners [3].

For a better prediction of location, Predictive Location Model (PLM) was proposed. This model deployed geometrical and topological techniques. Users are allowed to gain services on time and on demand. Predictive Location Model can improve performance to predict locations in Location Based Services (LBS) with granular road-level. This work applied map-based location prediction approach to obtain many advantages for calculation and presentation of on-road services [4].

A group of researcher studied some of their own work on location modeling, location updates, caching of location-dependent data, and batch processing of spatial queries. Then, they developed some prototypes of Location Based Services (LBS) which are applicable for real-life application. They applied some similar techniques as the traditional database e.g. data modeling, indexing, caching, and query optimization [5].

Location Based Services (LBS) was studied in a laboratory setting. This laboratory study was not affected by some real-world technical constraints e.g. slow response times or imprecise localization information. Without those previous constraints, they were able to focus on the main effects of LBS on users' perceptions, as well as efficiency gains. This study result showed that location-tracking and location-aware services are different in term of users' perspective. Location-tracking is easier and more useful for users. Location-aware seems not helpful for them. Users in this work exhibited distinctively concern for privacy issue while they were being examined by this study investigation [1].

An intelligent information master system was develop to serve in tourism industry. This system applied Location Based Services and Google maps. This system deployed a web service with an ontological system database to provide tourism information in particular of an archeological site in New Taipei City, Taiwan e.g. travelling route plan. This system can provide GPS function, QR and Barcode reader, and could server accessibility [6].

Suan Sunandha Rajabhat University (SSRU), Thailand deployed a lot of mobile applications to manage several academic activities and services i.e. a mobile application for internship program management. This mobile application creates multi communicational channels for SSRU student, management from hosting organization that adopts student as intern, and advisor of student to exchange vital data and information regarding performance and excellence of the internship program. Student's advisor can track location of students where they are having working practices through this application. Students cannot practice their working skills outside predefined working domains. Unless their supervisor or trainer verify that students are commanded or sent to work outside organization. This could be extra training located at training centers or onsite service dispatch that students have to deliver onsite service for real customers of the host organizations [7].

\section{Methodology}

\section{A. Google Cloud Platform (GCP)}

Google Cloud Platform provides cloud services through variety of physical assets e.g. data storage, computer hardware, computer network, virtual machines, data center and cloud server for Internet of Thing. Moreover, not just physical assets, Google Cloud Platform also provides developer tools and API services for developers to retrieve Google services via cloud. Developers can customize these services and create scenarios or tailor all services into combinations. Optimization of Google Cloud Platform interacts with relational regions and zones of developers. Geographical distribution of Google Cloud Platform would cause latency and poor performance when it is operated cross zone and region.

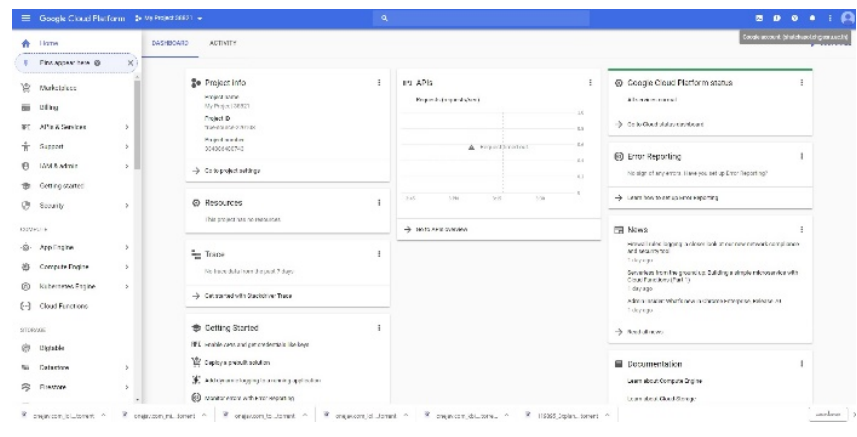

Fig. 1. Google Cloud platform console.

This main console is presented in a form of web-based user interfaces to let software developers interact with Google services and resources. Developers are enabled to manage several projects via this web-based console to access to Google APIs and services. Developers can deploy compute engines and storage service as well as APIs. Single and multiple project creation are viable on Google Cloud Platform Console. However, each project will separate its work for accessibility of each project team members to facilitate project.

\section{B. Google Map APIs}

Google application programming interfaces (APIs) is a set of communication protocol and components to communicate with Google Services. This work deployed Google Maps APIS to retrieve data of locations and routes Google Maps 
Platform. Android application that intends to deal with map, location and routes relies on Maps SDK for Android. This SDK automatically handles access to Google Maps servers via various types of APIs provided. Map display and navigation system are responsive to user gestures such as clicks, drags and zooms. This is a list of APIs that were implemented in this work: Directions API, Distance Matrix API, Maps Embed API, Geolocation API, and Places SDK for Android.

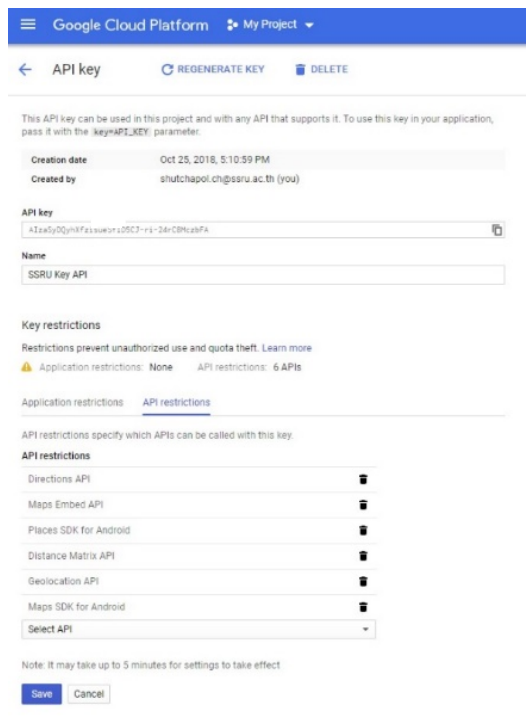

Fig. 2. Google Maps APIs Implemented in project.

Google Could Console shows which Google APIs were enabled in this project. APIs key for communication with Google Maps Platform was shown in Fig. 2. This string has be censored for security and privacy issues of this project. All implemented APIs were deployed in Android Studio to create map display, route finding, and real-time navigation on mobile screen. Developer embedded this APIs key in an Android Studio project as a connection string for Android application.

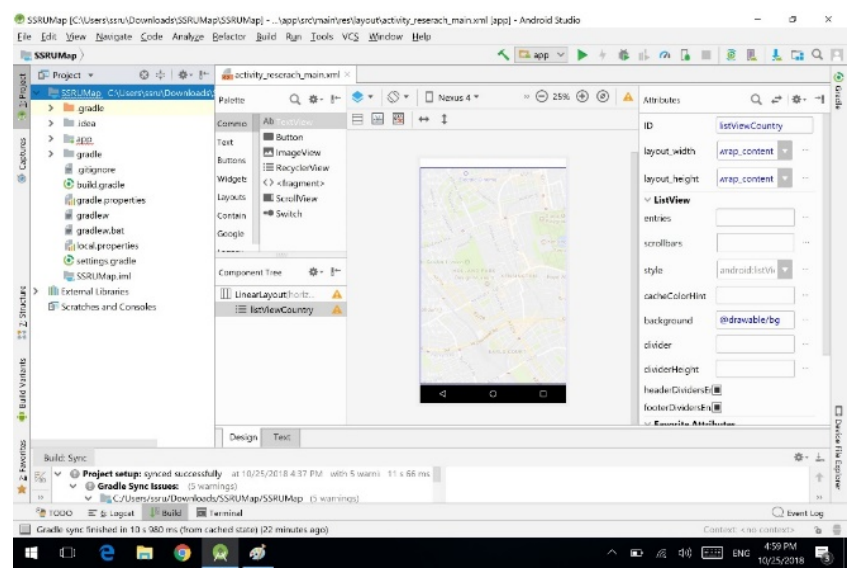

Fig. 3. Main map layout of Android application.

\section{RESUlts AND DisCUSSIONS}

This work applied Location Based Services (LBS) to provide information of map, places and navigation based on location of all buildings on the main campus of Suan
Sunandha Rajabhat University (SSRU), Thailand. All services delivered through an application run on user's mobile phones or smart phones with Android Operating System. Moreover, it also provides other relational information e.g. interior and exterior pictures of each building, contact information and links to each building website. Users are enabled to search each target building on campus by selecting pinpoint or marker one the map or lookup via search box menu. Once you define a starting point or current location at that time, later users have to specify a destination. Application will draw a navigation path from 2 places with the best route result. Users will be guided direction by compass arrow. User can zoom in or out current location on the map to compare it with the real location onsite or users can compare exterior pictures of each building with real vision onsite to verify a correct route. User can rely on this application without extra assistance from human or person nearby.

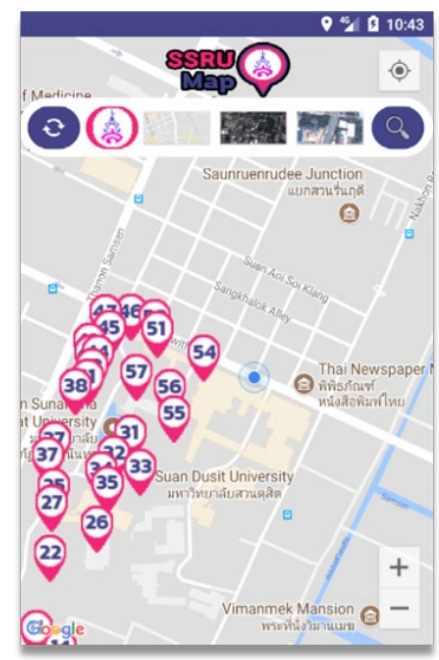

Fig. 4. Overview of pinpoints on map of all buildings.

Overview of SSRU map shows markers of major building while minor building markers are hidden in this view. However, users can enlarge this map by pinching gesture, zoom in to reveal all details. All major markers when users first open application were displayed in Fig. 4 with traffic mode view map. Default view of this application is traffic mode view and users can switch to other view e.g. satellite and hybrid mode on demand. Moreover, this application also displays other landscape of SSRU e.g. a small lake and a football field on the map.
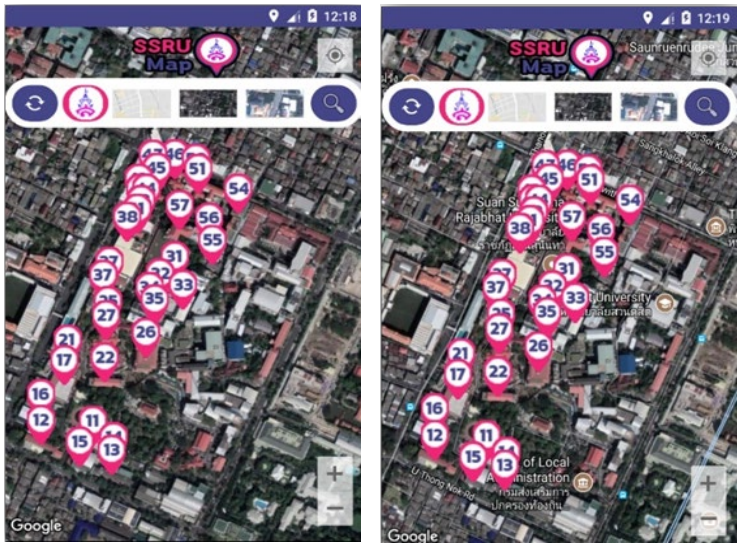

Fig. 5. Satellite and hybrid views available on this application. 
Most of the time, users are confused by a contrast view of traffic view. There are many users who prefer satellite or hybrid views to compare the current location and nearby places on the map. Hybrid view is a good practice to serve navigation feature of this application. While user is trying to detect current location on the map, users will see.

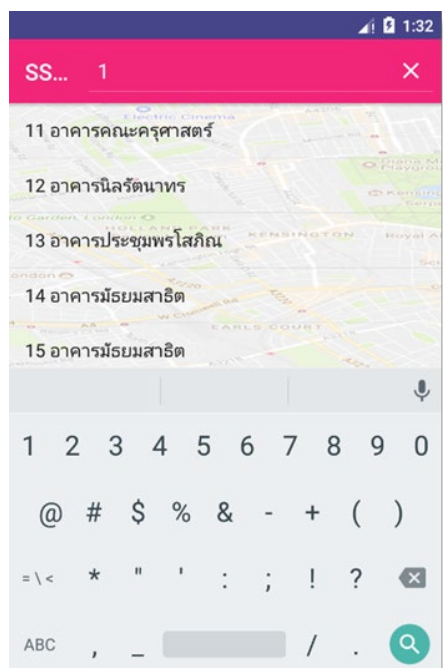

Fig. 6. Search menu box sorted by numeric order.

Users have 2 options to find a desirable place that they are looking for. First, they can pick up a pinpoint shown on the map or they can touch a search menu on top of this application. Users can lookup building information by defining building number and result will be listed and shown numerically. It is easy for visitors to call each building by its number because each building name is Thai traditional name and it is very long name.

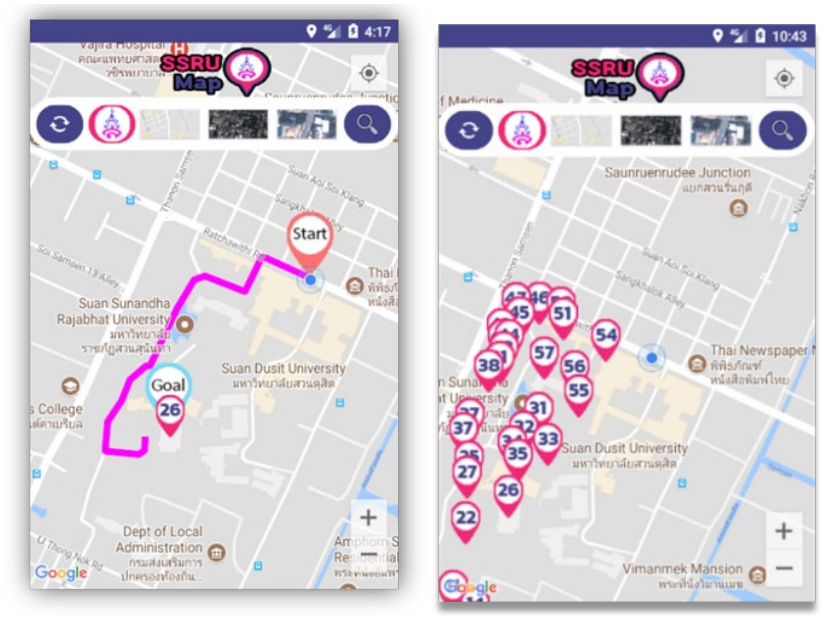

Fig. 7. Display of current location of user on map.

Users are enabled to track their moving direction while they are following a path suggested by application. Blue dot in the Fig. 6 indicates user's current location from a start point. User will be able to follow this path or move to other directions and a new path will be calculated and shown on screen. This feature will accompany user to reach out the destination without getting lost and wasting time.

\section{CONCLUSION}

This work applied Location Based Services framework on an Android mobile application called SSRU Map. This application deployed Google Map Platform to retrieve all features based on Location Based Services framework e.g. map, place, route, and direction of the main campus of Suan Sunandha Rajabhat University (SSRU), Thailand. This application is aimed to serve thousands of new visitors who come to SSRU every year for several purposes e.g. test taker, seminar and conference participant, tourist, and site visitor. All data, traffic, and statistics of users will be stored on Google Cloud Platform for further analysis i.e. which building is too difficult for visitors to find by themselves and they have to rely on this application solely. Moreover, geolocation data retrieved from this application for visitors will be studied for better understanding and well instructed design signal board, not to confuse visitors. Finally, university management team will be able to bring this study to specify advanced geology strategies for university.

\section{ACKNOWLEDGMENT}

This research was supported by Research and Development Institute, Suan Sunandha Rajabhat University.

\section{REFERENCES}

[1] I. A. Junglas and R. T. Watson, "Location-based services evaluating user perceptions of location-tracking and location-awareness services," Communication of the ACM, vol. 51, no. 3, pp. 65-69, 2008.

[2] C. Pontikakos, M. Sambrakos, T. Glezakos, and T. Tsiligiridis. (2018) Location-Based Services: A Framework for an Architecture Design. [Online]. Available: https://pdfs.semanticscholar.org/6113/ db271bc359036e4dd0760969e3bcebe234d0.pdf

[3] S. Steiniger, M. Neun, and A. Edwardes, "Foundations of Location Based Services," CartouCHe1-Lecture Notes on LBS V. 1.0, pp.1-28, 2006.

[4] H. A. Karimi and X. Liu, "A predictive location model for location-based services," in Proc. the Eleventh ACM International Symposium on Advances in Geographic Information Systems, 2003, pp.126-133.

[5] D. L. Lee, M. Zhub, and H. Hua, "When location-based services meet databases,” Mobile Information Systems, vol. 1, pp. 81-90, 2005.

[6] S. Y. Yang and C. L. Hsu, "A location-based services and Google maps-based information master system for tour guiding," Computers \& Electrical Engineering, vol. 54, pp. 87-105, 2016.

[7] S. Chopvitayakun, "Hybrid framework as a cross platform tool for designing and developing a mobile application serving the internship program management," International Journal of Management and Applied Science, vol. 5, no. 1, pp. 35-39, 2017.

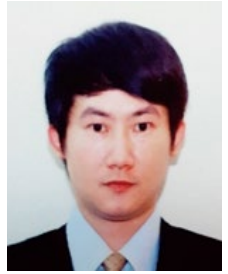

Shutchapol Chopvitayakun is with Information Technology Program, Faculty of Science and Technology, Suan Sunandha Rajabhat University, Bangkok, Thailand. 